\title{
Cloning and Expression of Truncated Chlamydial Major Outer Membrane Protein in E.coli: A Miniature Step Forward
}

\author{
Razieh Bitazar ${ }^{1,2}$, Taghi Naserpour Farivar ${ }^{1 *}$, Bahareh Hajikhani ${ }^{2 *}$, Rezvan Bagheri ${ }^{2}$ and Ali Salimi ${ }^{3}$ \\ ${ }^{1}$ Cellular and Molecular Research Center, Qazvin University of Medical Sciences, Qazvin, Iran \\ ${ }^{2}$ Reproductive Biotechnology Research Center, Avicenna Research Institute, ACECR, Tehran, Iran \\ ${ }^{3}$ Monoclonal Antibody Research Center, Avicenna Research Institute, ACECR, Tehran, Iran
}

\begin{abstract}
Administration of Prophylactic vaccines still is the cutting-edge of prevention, control and elimination of infections. Among the common causes of sexually transmitted diseases, chlamydia trachomatis is an important socioeconomic burden worldwide which impairs human genital tract function, resulting in severe complications like urethritis, pelvic inflammatory disease (PID), getting ease of HIV transmission, playing cofactor role in human papilloma virus (HPV)induced cervical neoplasia, and unfortunately infertility. Because of all difficulties, vaccination is considered to be the most economical and reliable way to escape from unrestrained exacerbation states of infection than any other prevalence program. But despite much advancement in the field of veterinary, the dream has yet to be realized. Among a number of potential candidates, the major outer membrane protein (MOMP) is a vanguard of subunit vaccines. In this attempt, MOMP217 gene fragment cloned in PET28b+ and expressed in E.coli. The protein expression confirmed by SDS-PAGE. These findings demonstrate that, considering the antigenicity prediction and due to the existence of potential epitopic region, rMOMP217 could be a potential capable peptide which can represent as an alternative to whole MOMP.
\end{abstract}

Keywords: Chlamydia trachomatis; MOMP (Major Outer Membrane Protein); Vaccine

\section{Introduction}

Worldwide hurricane of 92 million cases of chlamydia trachomatis infection was estimated by WHO in 2001 [1]. High prevalence of sexually transmitted infections in Europe [2] and in the United States [3] would recommend it as a leading cause of cervicitis, pelvic inflammatory disease (PID), Salpingitis, ectopic pregnancy and infertility [4]. It is too prevalent in developing countries [5]. Chlamydia as a mucosotropic [6] intracellular bacterium is a facilitating factor of HIV transmission and also is a cofactor of HPV-induced cervical neoplasia [7]. Thus, administration of vaccine absolutely is necessary to save $\$ 2$ billion per year [8]. The perfect protection model is focused on subunit vaccine using major outer membrane protein (MOMP) [9]. Among different proteins of chlamydial, MOMP as an immunodominant, SurfaceExposed antigen is mostly investigated as the best potentially candidate for a subunit vaccine. MOMP is greatly antigenic which known as a porin and the representative of Peptidoglycan layer [10]. In this attempt, beside molecular gene cloning and protein expression methods in prokaryotic system, antigenic domain of MOMP were predicted using prediction tools. Our suggestion is that MOMP160-376 which named rMOMP217 during this paper would be a new member of potentially vaccine candidate among collection investigated during so many years. But undoubtedly this present study needs further experimental works to develop an effective vaccine instead of both therapeutic and prophylactic proceedings.

\section{Material and Methods}

\section{Bacterial strains And Vector}

Chlamydia trachomatis strain L1/440, E.coli DH5a and E.coli BL21 (DE3) for cloning and Expression experiments were obtained from Avicenna Research Institute Microbial Bank. pET28b expression vector was also provided from Avicenna Research Institute.

\section{PCR Amplification}

MOMP217 was amplified with Taq DNA polymerase (Vivantis). Primers were designed with BamHI and HindIII restriction sites at the $5{ }^{\prime}$ of forward and reverse, respectively. Primers designed to amplify MOMP217 gene fragment (corresponding to nucleotides 160-376) was according to information retrived from NCBI data Bank. The forward primer incorporated a BamHI restriction enzyme site (bold) and a start codon (underlined) with the sequence, 5'-GTTGGGGATCCTGGAGATAATGAAAAT-3' and the reverse primer incorporated a HindIII site (bold) and a stop codon (underlined), 5'-ACTCTAAGCTTCTCAACTGTAACTGCGTATTT-3' (Figure 1). In brief Total volume of each reaction was $25 \mu \mathrm{l}$ which consisted of $1 \mathrm{mM}$ of each primer, 10X PCR buffer $0.6 \mathrm{mM}$ dNTPs, $5 \mathrm{mM} \mathrm{MgCl}$, $1.0 \mathrm{U}$ Taq polymerase (Vivantis) and $1 \mu \mathrm{l}$ of the template DNA. PCR condition were as follow: initial denaturing at $95^{\circ} \mathrm{C}$ for $5 \mathrm{~min}, 40$ cycles including $30 \mathrm{~s}$ at $95^{\circ} \mathrm{C}, 30 \mathrm{~s}$ at $53.5^{\circ} \mathrm{C}, 1 / 30 \mathrm{~s}$ at $72^{\circ} \mathrm{C}$ and a final extension at $72^{\circ} \mathrm{C}$ for $5 \mathrm{~min}$. Hence, the expected $674 \mathrm{bp}$ band was purified directly with clean-up process using Qiagen purification kit buffers.

*Corresponding author: Taghi Naserpour Farivar, Cellular and Molecular Research Center, Qazvin University of Medical Sciences, Qazvin, Iran, Tel: +98 281 3336002; +98 21 22432020; E-mail: Taghin@qums.ac.ir

Bahareh Hajikhani, Reproductive Biotechnology Research Center, Avicenna Research Institute, ACECR, Tehran, Iran, E-mail: B.Hajikhani@avicenna.ac.ir

Received December 21, 2013; Accepted January 25, 2014; Published January 28, 2014

Citation: Bitazar R, Farivar TN, Hajikhani B, Bagheri R, Salimi A (2014) Cloning and Expression of Truncated Chlamydial Major Outer Membrane Protein in E.coli: A Miniature Step Forward. J Vaccines Vaccin 5: 217. doi: 10.4172/2157-7560.1000217

Copyright: (C) 2014 Bitazar R, et al. This is an open-access article distributed under the terms of the Creative Commons Attribution License, which permits unrestricted use, distribution, and reproduction in any medium, provided the original author and source are credited. 


\section{Bioinformatic}

Amino acid sequence of MOMP in FASTA format was elicited from protein data bank. There is no available evidence from 3D structure of MOMP in PDB (Protein Data Bank). Prediction of antigenic domain of MOMP was performed by Kolaskar and Tonaonkar method.

Secondary structure of MOMP serovar L1 was predicted by GOR (Garnier-Osguthorpe-Robson). Multiple Alignments between serovar E, L1, L2 and L3 for detecting the conserved common regions was performed by European Bioinformatics Institute website.

\section{Cloning of PET-MOMP217}

PCR was performed to amplify the MOMP217 containing epitopic regions with specific primers. Qiagen gel extraction kit (Qiagen, Hilden, Germany) was used to purify PCR amplified product and plasmid DNA. Purified plasmid and amplified insert were double digested with BamHI and HindIII (Fermentase). The digested products were purified and ligated into linearised and purified vector withT4 DNA ligase enzyme(Fermentase), then ligation mixture were transformed into E.coli DH5a cloning host. As well, Recombinant clones were confirmed by colony PCR and double digestion. The confirmed purified DH5a/ pET- MOMP217 positive colony were transformed and cloned into E.coli BL21 (DE3) expression host and used for protein expression purpose with IPTG.

\section{Protein expression}

E.coli BL2 (DE3) with T7 promoter, harboring the PET- MOMP217 $(24 \mathrm{kDa})$ positive colony were grown in $100 \mathrm{ml} \mathrm{LB}$ broth at $37^{\circ} \mathrm{C}$ including $100 \mu \mathrm{g} / \mathrm{ml}$ kanamycin (Sigma) to achieve an optical density (OD) of 0.7 (stationary growth phase) at $600 \mathrm{~nm}$ at this time, $1 \mathrm{mM}$ isopropyl-ß-D-thiogalactoside (IPTG; Invitrogen) were added to $50 \mathrm{ml}$ $\mathrm{LB}$ broth and the other same amount were considered as uninduced (-IPTG) control. Incubation was continued at $37^{\circ} \mathrm{C}$. The samples from induced and uninduced culture were collected within 2, 4, 6 hours and overnight after induction. Incubation temperature in the case of overnight was reduced to $25^{\circ} \mathrm{C}$. Samples were accumulated, centrifuged at $6000 \mathrm{rpm}$ for $5 \mathrm{~min}$. Harvested cells were resuspending with $2 \mathrm{x}$ loading protein buffer containing 2-ME, boiled and subjected to SDS-

$10 \quad 20 \quad \cdot 30 \quad .40 \quad .50$

MKKLLKSVIVFAALSSASSLQALPVGNPAEPSLMIDGILWEGFGGDPCDP

epitope ++++++++++++++++++++++++++++ ++++++++++ ++++++

$60 \cdot 70 \cdot 80 \cdot 90 \cdot 100$

CTTWCDAISMRMGYYGDFVFDRVLQTDVNKEFQMGAKPTATTGNAAAPST

$\begin{array}{ccc}\text { epitope }++++++++ & +++++++++++ \\ 110 \quad .120 & .130 \quad .140 .150\end{array}$

CTARENPAYGRHMQDAEMFTNAAYMALNIWDRFDVFCTLGATSGYLKGNS epitope

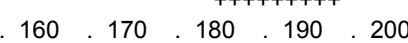

ASFNLVGLFGDNENQSTVKKDAVPNMSFDQSVVELYTDTTFAWSVGARAA

epitope +++++++ ++++++++ +++++++++

210.220 .230 .240 .250

LWECGCATLGASFQYAQSKPKVEELNVLCNAAEFTINKPKGYVGKEFPLD

epitope ++++++++++++++++++++++++++++++++++++ +++++++

260.270 .280 .290 .300

LTAGTDAATGTKDASIDYHEWQASLALSYRLNMFTPYIGVKWSRASFDAD epitope

$310 \quad 320 \quad 330.340 .350$

TIRIAQPKLATAIFDTTTLNPTIAGAGEVKANAEGQLGDTMQIVSLQLNK

epitope ++++++++++++ ++++++++

360.370 .380 .390

MKSRKSCGIAVGTTIVDADKYAVTVETRLIDERAAHVNAQFRF

epitope $++++++++++++++++++++++\quad+++++++$

Table 1: Prediction of Antigenic Region of Chlamydia Trachomatis Serovar L1.
PAGE analysis. Total protein expression trend were evaluated by $10 \%$ SDS-PAGE stained with Comassie Brilliant Blue. SDS-PAGE were shown to have one band corresponded to recombinant protein which were termed as rMOMP217 (Table 1).

\section{Result}

\section{Recombinant plasmid}

The MOMP217=truncated omp1, was amplified by polymerase chain reaction. Then, digested insert and linearised plasmid associated, pET28b, were ligated and cloned into E.coli DH5a cloning host resulting DH5a /PET-MOMP217 Recombinant clones were transferred into BL21 expression host concluding BL21/PET-. MOMP217.

\section{Expression of recombinant protein}

The expression of rMOMP217 was induced by adding IPTG in $1 \mathrm{mM}$ final concentration. The mass of rMOMP in induced and uninduced culture were evaluated by SDS-PAGE. The $24 \mathrm{kDa}$ molecular mass was not noticeable in uninduced cells whereas was recognizable within 2,4 , 6 hours and over night after induction (Figure 2).

\section{Bioinformatic and antigenicity prediction research}

The multiple alignments of the MOMP sequences by clustalw2 helped to determine conserved common regions between L1, L2, L3 and $\mathrm{E}$ serovars respectively. This experiment showed high similarity in conserved domain. C.Trachomatis serovar L1 was as a template in the entire of study. Crucial attributes of antigenic epitopes are flexibility and hydrophilicity; secondary structure prediction by GOR shown flexible region like random coil. Antigenicity prediction by Kolaskar and Tonaonkar method demonstrated antigenic domain which were shown by plus (Table 1 ).

\section{Discussion}

Is Vaccinology almost forgotten? To be frank, neither screening programs nor controlling trials are able to overcome the threats about reproductive issue. Whereas, Vaccination is unequivocally pivotal to tackle the diseases. It has also been one of the most important health advances in history. Approximately, 92 million young adults suffer from this considerable public health concern [11]. Therefore, vaccination is an important strategy not only for chlamydiosis but also for other STIs [12]. Complications like ectopic pregnancy, pelvic inflammatory disease (PID) and infertility suggest more attention on this turbulent, silent and unfortunately neglected disease. Particularly, for women lack of a protective vaccine has existed as the health problem. Data were gathered from multiple sources at various time points noticed the importance of MOMP as a subunit vaccine candidate. MOMP, encoded by OmpA gene, has been the antigen tested in many investigational studies and known as a primary of vaccine development [13]. MOMP is a promising, immunodominant and Surface-Exposed antigen which is able to stimulate both humoral and cellular immunity [14]. To date, no vaccine licensed for human use is available. This view is supported by A. Feher who writes "a prophylactic vaccine has long been sought against C.trachomatis but has yet to be obtained" [15]. Moreover, despite advancement in the field of veterinary currently there is, however, no human protective vaccine [16]. Also, orientations of newly strategies in "OMICS" technology like vaccinomics are trying to produce an effective vaccine that would serve immunity against genital chlamydiosis [17]. In the history of vaccine development, single or multisubunit vaccine is the major focus of chlamydial Vaccinology [18]. Harmful effect of whole organism, as well reverting to a virulent form has shifted in 
subunit vaccine toward the first generation vaccine [19]. Despite the necessity of additional factors like delivery system and adjuvant [15], for both immunocompromised patients and pregnant women who can't safely take live vaccinations it would be an effective alternative. Human subunit vaccines are currently available against pneumococci, meningococci, Haemophilus influenza, and hepatitis B and influenza virus [20]. But to improve the insight into designing an effective vaccine, it is crucial to get concrete knowledge about immunological aspect of this infection, besides the role of both humoral and cellular immunity as two arms of immune system. Indeed, successful protection requires the stimulation of T-cell in addition to antibody responses [21]. In the current study, we were being able to predict precisely antigenic region through bioinformatic and antigenicity prediction tools to produce a truncated protein instead of a full-length. Truncated protein's nature reflecting this reality that short segment derive from protein make it more appropriate to fuse with other genes to construct a fusion protein. Not long ago, novel B-cell epitopes containing mostly conserved region and high antigenicity. They were MOMP73-81, MOMP161-175, MOMP217-225, MOMP261-270 and MOMP377-386 in the N-terminal region. Surprisingly MOMP377-386 was the strongest epitope followed by challenging in mice [22]. It is undoubtedly true that B-cell responses are essential. However, T-cell derived immune response and peptides including T-cell epitopes due to the significant role of CTL and Th during infection are absolutely important. Also, the small fragment of MOMP containing T-cell epitope, rMOMP278, was able to elicit both systemic, mucosal and a balanced Th1/Th2 immune responses [23]. It would be a valuable candidate in order to formulate a T-cell vaccine. Regarding to our research, MOMP217 were expressed as 6xHis-tagged protein. SDS-PAGE analysis confirmed desired expression condition and showed that this expressed protein could readily purify with $\mathrm{Ni}$ NTA affinity chromatography. To construct a recombinant plasmid, pET28b were used as an expression vector. This approach has an attractive feature that during protein purification, pET28b needs Thrombin to remove fusion tag, while other like pET32a resulting Trx-His-tag needs one more expensive enzyme like Enterokinase to remove the fusion tag. The mentioned fusion tag make an intervention in protein activity; obviously whenever total protein inject to animals, antibodies would elicit against both targeted protein and fusion tag. Thus, purification of protein in prokaryotic expression system with purification affinity tag like 6-His tag is easier than other vectors. It has a little influence on protein function because 6-His belongs to the insert amino acids with poor immunogenicity and is easy to remove [24]. The highest throughput of protein expression obtained at $30^{\circ} \mathrm{Cto}$ produce recombinant Hemoglobin II from Lucina pectinata [25]. A recent study optimized the convenient condition for mature PsaA expression in Escherichia coli for about $16 \mathrm{~h}$ at $25^{\circ} \mathrm{C}$ with $0.1 \mathrm{mM}$ IPTG in Terrific Broth medium [26]. Taken together, these findings have tended to focus on optimal condition for protein expression. Growth in $37^{\circ} \mathrm{C}$ resulted

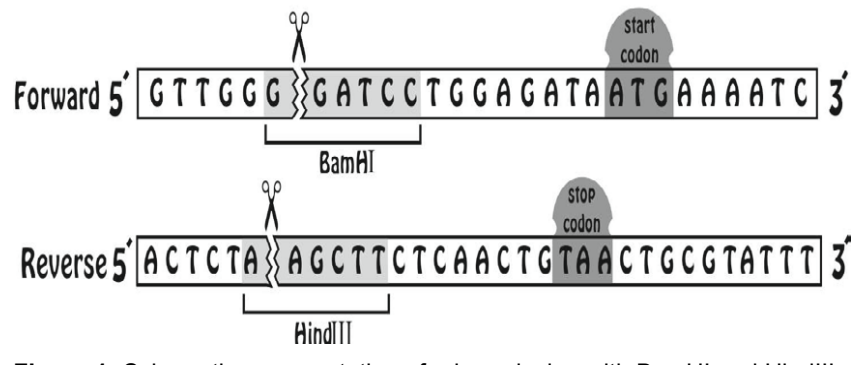

Figure 1: Schematic representation of primer design with BamHI and HindIII.

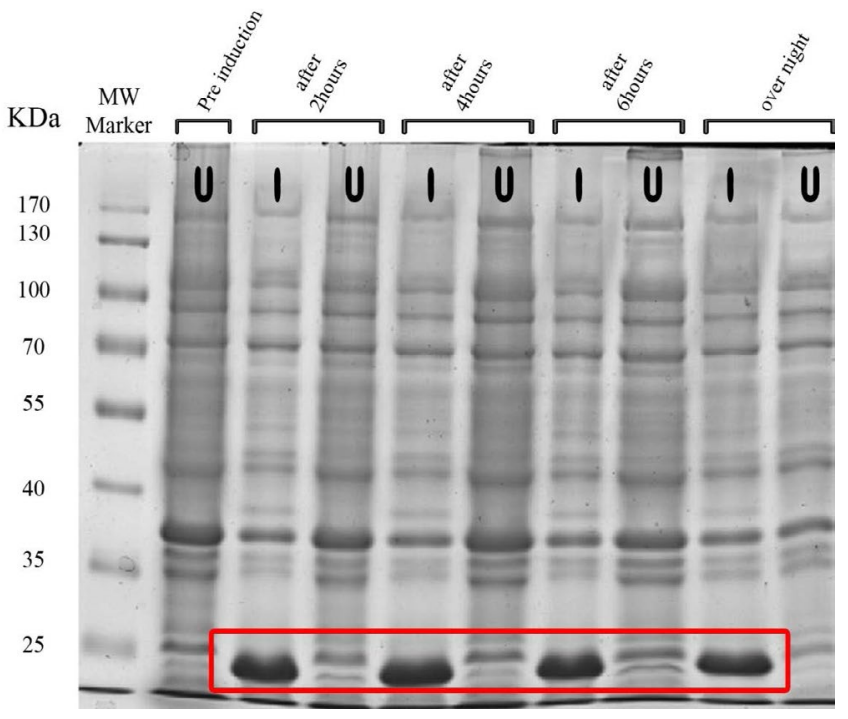

Figure 2: SDS-PAGE analysis of MOMP216 at different induction time. U: uninduction (-IPTG)

I: Induction (+IPTG) restriction sites.

in accumulation of inclusion body while incubation in $25^{\circ} \mathrm{C}$ produce more soluble form of protein, in fact this temperature helps the bacteria to consume all energy toward protein biosynthesis and not cell division. It may be explained by the fact that night expression without any needs to follow is the best system to increase the yield of protein expression. To our knowledge, the truncated protein in this study is a first report and contains some important epitopic region. The result for SDS-PAGE demonstrate that our constructed prokaryotic system PET28b-BL21 MOMP217 (DE3) produce target recombinant protein. The current investigation was limited by economical problem as a big issue in developing countries. Accordingly, more research on this topic needs to be attempted before introducing as a vaccine candidate. Therefore, further empirical researches on the current study are recommended. Since, massive improvement is needed in human vaccine, recent developments have heightened a convenient adjuvant and delivery system for a human vaccine. The vaccine needs to promote two CMI and antibody response, CAF01 is an adjuvant that is appropriate to enter human clinical trials in future [27]. CPG and Montanide are two other adjuvants that are now in clinical trials $[28,29]$. In addition, Novel delivery systems, including Vibrio cholerae ghosts and cationic liposomes, may have the potential to elicit protective responses against chlamydial genital infection when used in conjunction with appropriate antigens [30]. To summarize, if the debate is to move forward, a better understanding of more delivery systems and eligible adjuvant needs to be developed. As well, better technological possibilities combined with proteomics, immunology and immunoinformatic, allow for new vaccination strategies (Figures 1 and 2).

\section{Acknowledgement}

Financial Support and help from Dr.Ali Bitazar is gratefully acknowledged. We also thank Dr. Keivan Tadayon and Mr.Mohammad Sekhavati from Raz vaccine serum and research institute of Iran for his helpful guidance in manuscript publishing process.

\section{References}

1. WHO (2012) Global Prevalence and Incidence of selected Curable Sexually Transmitted Infections: overview and estimates. Geneva, Switzerland.

2. Kløvstad H, Natås O, Tverdal A, Aavitsland P (2013) Systematic screening with 
Citation: Bitazar R, Farivar TN, Hajikhani B, Bagheri R, Salimi A (2014) Cloning and Expression of Truncated Chlamydial Major Outer Membrane Protein in E.coli: A Miniature Step Forward. J Vaccines Vaccin 5: 217. doi: 10.4172/2157-7560.1000217

information and home sampling for genital Chlamydia trachomatis infections in young men and women in Norway: a randomized controlled trial. BMC Infect Dis 13: 30 .

3. Wang ZY, Fu GY, Wang SM, Qin DC, Wang ZQ, et al. (2013) Rapid screening for Chlamydia trachomatis infection by detecting Ît-mannosidase activity in urogenital tract specimens. BMC Infect Dis 13: 36.

4. Kohli R, Konya WP, Obura T, Stones W, Revathi G (2013) Prevalence of genital Chlamydia infection in urban women of reproductive age, Nairobi, Kenya. BMC Res Notes 6: 44.

5. Silva LC, Miranda AE, Batalha RS, Sabino CC, Dib E, et al. (2012) Chlamydia trachomatis infection among HIV-infected women attending an AIDS clinic in the city of Manaus, Brazil. Braz J Infect Dis 16: 335-338.

6. Murthy AK, Guentzel MN, Zhong G, Arulanandam BP (2009) Chlamydial protease-like activity factor--insights into immunity and vaccine development. J Reprod Immunol 83: 179-184.

7. Yu H, Jiang X, Shen C, Karunakaran KP, Brunham RC (2009) Novel Chlamydia muridarum $T$ cell antigens induce protective immunity against lung and genital tract infection in murine models. J Immunol 182: 1602-1608.

8. Hu D, Hook EW 3rd, Goldie SJ (2004) Screening for Chlamydia trachomatis in women 15 to 29 years of age: a cost-effectiveness analysis. Ann Intern Med 141: $501-513$.

9. Pal S, Theodor I, Peterson EM, de la Maza LM (2001) Immunization with the Chlamydia trachomatis mouse pneumonitis major outer membrane protein can elicit a protective immune response against a genital challenge. Infect Immun 69: 6240-6247.

10. Wang Y, Berg EA, Feng X, Shen L, Smith T, et al. (2006) Identification of surface-exposed components of MOMP of Chlamydia trachomatis serovar F. Protein Sci 15: 122-134.

11. Farris CM, Morrison SG, Morrison RP (2010) CD4+ T cells and antibody are required for optimal major outer membrane protein vaccine-induced immunity to Chlamydia muridarum genital infection. Infect Immun 78: 4374-4383.

12. Hofstetter AM, Rosenthal SL (2013) Health care professional communication about STI vaccines with adolescents and parents. Vaccine

13. Cai S, He F, Samra HS, de la Maza LM, Bottazzi ME, et al. (2009) Biophysical and stabilization studies of the Chlamydia trachomatis mouse pneumonitis major outer membrane protein. Mol pharm 6: 1553-1561.

14. Cambridge CD, Singh SR, Waffo AB, Fairley SJ, Dennis VA (2013) Formulation, characterization, and expression of a recombinant MOMP Chlamydia trachomatis DNA vaccine encapsulated in chitosan nanoparticles. Int J Nanomedicine 8: 1759-1771.

15. Feher VA, Randall A, Baldi P, Bush RM, de la Maza LM, et al. (2013) A 3-dimensional trimeric $\left.\right|^{2}$-barrel model for Chlamydia MOMP contains conserved and novel elements of Gram-negative bacterial porins. PLoS One 8: e68934.

16. Igietseme JU, Eko FO, Black CM (2011) Chlamydia vaccines: recent developments and the role of adjuvants in future formulations. Expert Rev Vaccines 10: 1585-1596.

17. Brunham RC, Rappuoli R (2013) Chlamydia trachomatis control requires a vaccine. Vaccine 31: 1892-1897.

18. Carey AJ, Beagley KW (2010) Chlamydia trachomatis, a hidden epidemic effects on female reproduction and options for treatment. Am J Reprod Immunol 63: $576-586$
19. Schautteet K, De Clercq E, Vanrompay D (2011) Chlamydia trachomatis vaccine research through the years. Infect Dis Obstet Gynecol 2011: 963513.

20. Lu C PB, Li Z, Lei L, Li Z, Chen L, et al. (2013) Induction of protective immunity against Chlamydia muridarum intravaginal infection with the chlamydia immunodominant antigen macrophage infectivity potentiator. Microbes Infect 15: $329-338$

21. Hafner LM, Wilson DP, Timms P (2013) Development status and future prospects for a vaccine against Chlamydia trachomatis infection. Vaccine

22. Shanli Zhu JC, Meixia Zheng, Wenci Gong, Xiangyang Xue, Wenshu Li, et al. (2010) Identification of immunodominant linear B-cell epitopes within the major outer membrane protein of Chlamydia trachomatis. Acta biochimica et biophysica Sinica 42: 771-778.

23. Murtada A Taha SRS, Kara Hulett, Shreekumar R. Pillai, Ronald Agee, et al. (2011) Peptide Containing T-Cell Epitopes of Chlamydia trachomatis Recombinant MOMP Induces Systemic and Mucosal Antibody Responses in Mice. World Journal of Vaccines 138-147.

24. Yang P, Wang KZ, Shi ZB, Dang XQ, Yu PB, et al. (2006) Prokaryotic expression, purification and activity assay of recombinant vascular endothelial growth factor. Nan fang yi ke da xue xue bao 26: 1263-1268.

25. Ramos C PR, Lorenzo W, Roman E, Granell LB, Cadilla CL, et al. (2010) Recombinant hemoglobin II from Lucina pectinata: a large-scale method for hemeprotein expression in E. coli. Protein J $29: 143-151$.

26. Larentis AL AA, Esteves Gdos S, Jessouron E, Galler R, Medeiros MA, et al. (2011) Cloning and optimization of induction conditions for mature PsaA (pneumococcal surface adhesin A) expression in Escherichia coli and recombinant protein stability during long-term storage. Protein expression and purification. Protein Expr Purif 78: 38-47.

27. Hansen J, Jensen KT, Follmann F, Agger EM, Theisen M, et al. (2008) Liposome delivery of Chlamydia muridarum major outer membrane protein primes a Th1 response that protects against genital chlamydial infection in a mouse model. $J$ Infect Dis 198: 758-767.

28. Pal S, Peterson EM, de la Maza LM (2005) Vaccination with the Chlamydia trachomatis major outer membrane protein can elicit an immune response as protective as that resulting from inoculation with live bacteria. Infect Immun 73 8153-8160.

29. Hirunpetcharat CWJ, Sakkhachornphop S, Nitkumhan T, Zheng YZ, Pichyangku $S$, et al. (2003) CpG oligodeoxynucleotide enhances immunity against bloodstage malaria infection in mice parenterally immunized with a yeast-expressed $19 \mathrm{kDa}$ carboxyl-terminal fragment of Plasmodium yoelii merozoite surface protein-1 (MSP1(19)) formulated in oil-based Montanides. Vaccine 21: 21-22.

30. Agger EM, Rosenkrands I, Hansen J, Brahimi K, Vandahl BS, et al. (2008) Cationic liposomes formulated with synthetic mycobacterial cordfactor (CAF01): a versatile adjuvant for vaccines with different immunological requirements. Plos one 3: e3116. 